\title{
Blending Laws for High-Molecular-Weight Polymer Melts
}

\author{
W. M. Prest, Jr.* \\ Polymer Science and Engineering, Universisy of Massachusetts, \\ Amherst, Mass. 01002, U.S. A.
}

(Received March 11, 1972)

\begin{abstract}
Blending laws have been previously proposed to describe the effects of molecular weight distribution (MWD) on the viscoelastic properties of polymer melts. The blending laws represent the relaxation spectrum of a blend $H_{\mathrm{B}}(\tau)$ as a weighted sum of the relaxation spectra of the individual components, each shifted in time by an amount characteristic of the interactions between the molecular species. In general, the binary blending law is
\end{abstract}

$$
H_{\mathrm{B}}(\tau)=\sum_{i, j=1}^{2} V_{i j} H_{i j}\left(\tau / \lambda_{i j}\right)
$$

The weighing factor $V_{i j}$, and shift factors $\lambda_{i j}$ may be obtained directly from the calculated relaxation spectra of blends of narrow MWD fractions. These parameters are used to test the applicability of two types of blending laws: (1) The quadratic blending law, $V_{i j}=w_{i} w_{j}$, where $w_{l e}$ is the weight fraction of the $k$-th component; (2) The simple blending law, $V_{i j}=v_{i} \delta_{i j}$. The experimental results give qualified support to the quadratic blending law but place strong restrictions on the magnitude and shape of the cross relaxation spectra term $H_{i j}$. These require that $H_{i j} \neq H_{j i}, H_{i j} / H_{i i}=$ constant, and $\lambda_{i j}=\lambda_{i i}$. In addition, the restrictions imply that the simple and the quadratic blending laws are equivalent representations of $H_{\mathrm{B}}(\tau)$.

KEY WORDS Blends / Blending Laws / Molecular Weight Distribution / Viscoelasticity / Relaxation Spectra /

Blending laws have been previously proposed ${ }^{1-3}$ to describe the effects of molecular weight distribution (MWD) on the viscoelastic properties of polymer melts. The blending laws represent the relaxation spectrum of a blend $H_{\mathrm{B}}(\tau)$, as a weighted sum of the relaxation spectra of the individual components, each shifted in time by an amount characteristic of the interactions between the molecular species.

Ninomiya ${ }^{1}$ originally suggested the simple blending law given by eq 1 .

$$
H_{\mathrm{B}}(\tau)=\sum_{i} v_{i} H_{i}\left(\tau / \lambda_{i}\right)
$$

where $v_{i}$ is the volume fraction of the $i$-th component and $H_{i}\left(\tau / \lambda_{i}\right)$ is the relation spectrum of that component shifted in time by a factor $\lambda_{i}$. This blending law has been examined in detail by Ninomiya, Ferry, and coworkers ${ }^{4-8}$ and has been used to predict the viscoelastic response,

* Present address: Rochester Corporate Research Laboratory, Xerox Corporation, Rochester, New York, N. Y., 14603, U.S.A. the zeroshear viscosity $\eta_{0 \mathrm{~B}}$, and the steady state shear compliance $J_{\mathrm{e} B}^{\circ}$ of several homopolomer blends and polymer-solvent systems.

Recently, it has been shown $\mathrm{n}^{9-12}$ that eq 1 does not explain the MWD dependence of $J_{\mathrm{e} B}^{\circ}$ and the viscoelastic response of high-MW polymer blends. Prest $^{9}$ has reported that the properties and response of high-MW poly(dimethylsiloxane) blends may be described by eq 1 with effective weighting factors $v_{i}$, which are in general less than the volume fractions of the components. Masuda and coworkers ${ }^{10}$ suggested that, in highmolecular-weight blends, entanglement couplings between the different MW species give rise to a set of relaxation mechanisms with time constants between the shifted relaxation times of the individual components. Bogue, et al., ${ }^{2}$ formalized this proposal by suggesting a quadratic blending law of the form

$$
\begin{aligned}
H_{\mathrm{B}}(\tau)= & w_{1}{ }^{2} H_{11}\left(\tau / \lambda_{11}\right)+2 w_{1} w_{2} H_{12}\left(\tau / \lambda_{12}\right) \\
& +w_{2}{ }^{2} H_{22}\left(\tau / \lambda_{22}\right)
\end{aligned}
$$


where $w_{i}$ is the weight fraction of the $i$-th component and $H_{12}(\tau)$ is a cross relaxation spectrum term. Recentley Graessley ${ }^{3}$ has proposed a theory to explain the effects of MWD in high-MW systems. This approach to binary blends considers the relaxation processes of molecule $A$ interacting with molecule $B$, to be different from those of B interacting with A. This leads to a quadratic blending law in which the cross term consists of the separate components $w_{1} w_{2} H_{12}\left(\tau / \lambda_{12}\right)$ and $w_{2} w_{1} H_{21}\left(\tau / \lambda_{21}\right)$, where in general $H_{12}(\tau) \neq H_{21}(\tau)$ and $\lambda_{12} \neq \lambda_{21}$.

A general binary blending law encompassing all of the above can be written as

$$
H_{\mathrm{B}}(\tau)=\sum_{i, j=1,2} V_{i j} H_{i j}\left(\tau / \lambda_{i j}\right)
$$

For the simple blending law $V_{i j}=v_{i} \delta_{i j}$ where $\delta_{i j}=1$ if $i=j$ and is zero otherwise and $v_{i}$ is the effective weighting factor, which is equal to the volume fraction for the Ninomiya blending law. The quadratic blending law is given by $V_{i j}=w_{i} w_{j}$ where $w_{k}$ is the weight fraction of each component.

The viscoelastic properties of a blend described by eq 3 may be calculated from the phenomenological relations of linear viscoelasticity ${ }^{13,14}$. The loss modulus of the blend $G_{\mathrm{B}}{ }^{\prime \prime}(\omega)$, is

$$
G_{\mathrm{B}}{ }^{\prime \prime}(\omega)=\sum_{i, j} V_{i j} G_{i j}^{\prime \prime}\left(\omega \lambda_{i j}\right)
$$

A similar equation applies to the storage modulus, $G_{i j}^{\prime}\left(\omega \lambda_{i j}\right)$. The properties of the blend in the terminal region, $J_{\mathrm{eB}}^{\circ}$ and $\eta_{\mathrm{OB}}$ are related to the properties of the component by

$$
\eta_{0 \mathrm{~B}}=\sum_{i, j} V_{i j} \lambda_{i j} \eta_{0 i j}
$$

and

$$
J_{\mathrm{eB}}^{\circ}=\sum_{i, j} V_{i j}\left(\lambda_{i j} \eta_{0 i j} / \eta_{0 \mathrm{~B}}\right)^{2} J_{\mathrm{e} i j}^{\circ}
$$

The experimental viscoelastic response of blends of different molecular weight polymers can be used to evaluate the parameters $V_{i j}$ and $\lambda_{i j}$. Previously these parameters were obtained by an intercomparison of the measured viscolastic properties, $G^{\prime}$ and $G^{\prime \prime}$, from eq 4 and related equations. However, this technique depends primarily on the choice of the poorly defined quantity $V_{22}$. A more direct test of the successes and deficiencies of the proposed blending laws can be obtained through a comparison of the calculated relaxation spectra of blends, $H_{\mathrm{B}}(\tau)$. This study determines the blending law parameters from relaxation spectra calculated from the previously reported ${ }^{12}$ viscoelastic response of blends of high-MW, narrow-MWD polystyrene samples. The experimentally defined parameters are used to analyze the proposed blending laws.

\section{EXPERIMENTAL}

\section{Relaxation Spectra}

Several approximations ${ }^{14}$ have been used to calculate $H(\tau)$ from the experimentally measured viscoelastic response. These techniques, involving various combinations of the derivatives of a viscoelatic function, are very sensitive to small variations in the data. In a companion study ${ }^{15}$, a comparison was was made between the relaxaspectra calculated by five different approximations. The method proposed by Tschoeg $1^{16}$. was found to produce the least fluctuations in the terminal region of the calculated $H(\tau)$. However, small fluctuations were apparent when the slope of $H(\tau)$ changed rapidly. Previous studies ${ }^{12}$ have shown that changes in MWD primarily effect the long time region of the viscoelastic response. The relaxation spectra presented in this paper were calculated by Tscoegl's approximation in order to examine the effects of MWD in the terminal region. The calculation of $H(\tau)$ is based on values of $G^{\prime}$ and $G^{\prime \prime}$ defined by the master curves of the samples viscoelastic response. The spectra determined from $G^{\prime}$ and $G^{\prime \prime}$ were in close agreement, indicating that both sets of data were consistant with each other.

The relaxation spectra at $T=192^{\circ} \mathrm{C}$, of blends formed by mixing samples with MWs of 97200 and 411000 , are shown in Figure 1. The numbers indicate the weight fraction of the high-molecularweight component. The relaxation spectrum of the $411000 \mathrm{MW}$, narrow-MWD component shows a long plateau at short and intermediate times. This is associated with the relaxation processes involving the interactions or "entanglements" betwen molecules. Others report a minimum in $H(\tau)$ in this region for high-MW systems with narrow MWD's ${ }^{10}$. The data presented here indicate a slight decrease in $H(\tau)$. This difference may be the result of the particular MWD. of the samples. However it should be noted. 


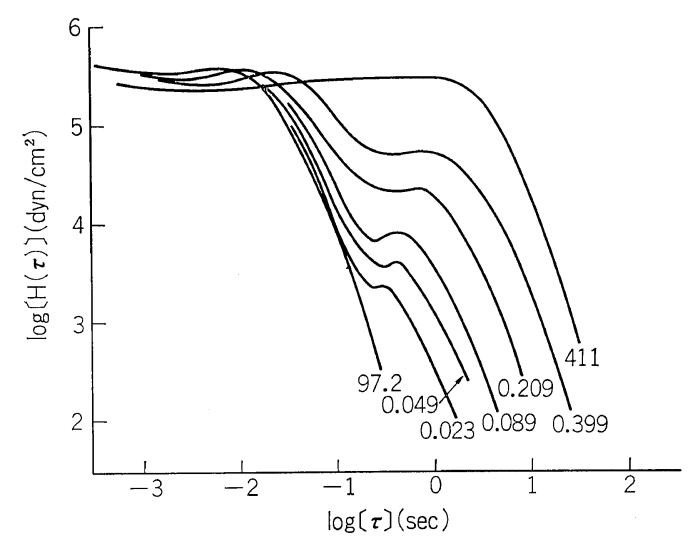

Figure 1. The relaxation spectra of blends of 97200-MW and 411000-MW polystyrene at $192^{\circ} \mathrm{C}$. The numbers indicate the weight fractions of the 411000-MW sample.

that the subsequent analysis of the data is insensitive to the specific shape of this intermediate response region of $H(\tau)$. At long times there is a very rapid decrease in $H(\tau)$. The slope of the $\log H(\tau) v s . \log \tau$ plot in the terminal region is initially equal to -2 and then becomes less than -3 . This end result, which is expected to be different from the $-1 / 2$ predicted by the Rouse theory ${ }^{17}$ for non-interacting molecules, is still much less than the -1 dependence predicted by the Chompff and Duiser theory ${ }^{18}$ for a network with mobile cross-links. The relaxation spectrum of the $97200 \mathrm{MW}$, narrow-MWD component also shows the same rapid decrease in $H(\tau)$ at long times and a short rubbery plateau, $50 \%$ higher than that observed for the 411000 MW sample.

The addition of a small amount of the 411000MW sample to the $97200-M W$ sample shifts the relaxation spectrum of the latter toward longer times and produces a distinct shoulder on $H(\tau)$. This shoulder becomes more prominant and greater in magnitude as the concentration of the high-MW component increases. Little significance can be attached to the small maxima which appear on the shoulders of the calculated $H_{\mathrm{B}}(\tau)$. These occur in a region where the numerical approximations for the second derivatives of VE functions used in the Tschoegl approximation are very sensitive to the experimental data ${ }^{15}$. Thus the calculated $H_{\mathrm{B}}(\tau)$ is

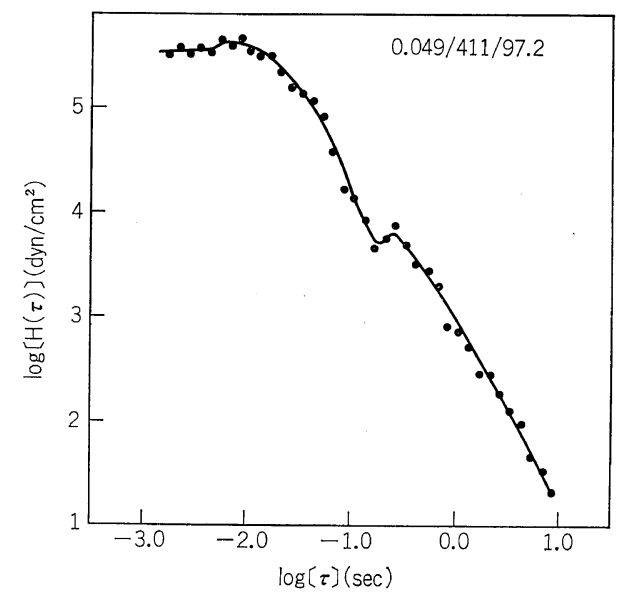

Figure 2. Relaxation spectrum of the $w_{2}=0.049$ blend.

expected to be subject to fluctuations. Typical oscillations in the experimental data are given in Figure 2 for the $w_{2}=0.049$ blend. The additional contribution to $H(\tau)$, represented by the shoulders, is responsible for the very high compliances of the blends. As $w_{2}$ increases, the initial fall-off in $H_{\mathrm{B}}(\tau)$ moves gradually to longer times while, beyond the shoulder, the final decrease in $H_{\mathrm{B}}(\tau)$ shifts rapidly towards much longer times.

\section{Parameters of the Simple Blending Law}

The general properties of the simple blending laws may be seen by considering the addition of idealized box shaped relaxation spectra. This is demonstrated in Figure 3 where $H_{i j}$ is the magnitude of the relaxation spectrum and $\tau_{i j}$ is the maximum relaxation time of component $i$ in blend $j$. By definition the weighting factors and shift parameters for blend $k$ are

$$
\begin{aligned}
& V_{11}=\frac{H_{1 k}-H_{2 k}}{H_{11}} \tau<\tau_{1 k} ; \lambda_{11}=\tau_{1 k} / \tau_{11} \\
& V_{22}=H_{2 k} / H_{22} \tau>\tau_{l k} ; \lambda_{22}=\tau_{2 k} / \tau_{22}
\end{aligned}
$$

Note that $V_{11}$ is determined by subtracting the contribution of $H_{2 k}$ from the relaxation spectrum of the blend, $H_{i k}$.

The calculated relaxation spectra shown in Figure 1 allow the direct measurement of the weighting factors $V_{i i}$ and the shift factors $\lambda_{i i}$ of the simple blending law. The contribution of the high-MW component to the relaxation 
spectrum of the blend $V_{22}$, is the ratio of the height of the shoulder of $H_{\mathrm{B}}(\tau)$ to the height of the plateu of the relaxation spectrum of the highMW component, $H_{22}(\tau)$. The shift factor $\lambda_{22}$ is the constant needed to superimpose the long-time portion of $V_{22} H_{22}(\tau)$ on $H_{\mathrm{B}}(\tau)$. The quantity $V_{11}$ is obtained from the low $\tau$ plateau response of the components by eq 7 . The shift factor $\lambda_{11}$ is determined by matching the initial decrease in the response of $H_{11}(\tau)$ and $H_{\mathrm{B}}(\tau)$. These values are listed in Table I.

In Figure 1, it appears that the shape of the long-time response changes with composition. This would make superposition an arbitrary procedure. However, close inspection of Figure 1 reveals that the apparent differences reflect the change in the shape of the terminal region of the high-MW components $H(\tau)$ from the intial $\tau^{-2}$ dependence to the final $\tau^{-3}$ response.

The terminal properties of the blends, $\eta_{0 B}$ and $J_{\mathrm{eB}}^{0}$, may be calculated from the blending law parameters with eq 5 and 6 or by the direct integration of $H_{\mathrm{B}}(\tau)$ according to the phenomeno- logical relations of linear viscoelasticity ${ }^{13,14}$. Table II demonstrates the agreement that is found between the calculated value of these properties and the experimental results.

\section{Weighting Factors}

The dependence of the weighting factors $V_{i i}$, on the respective weight fractions $w_{i}$ are shown in Figure 4. The brackets on the data are

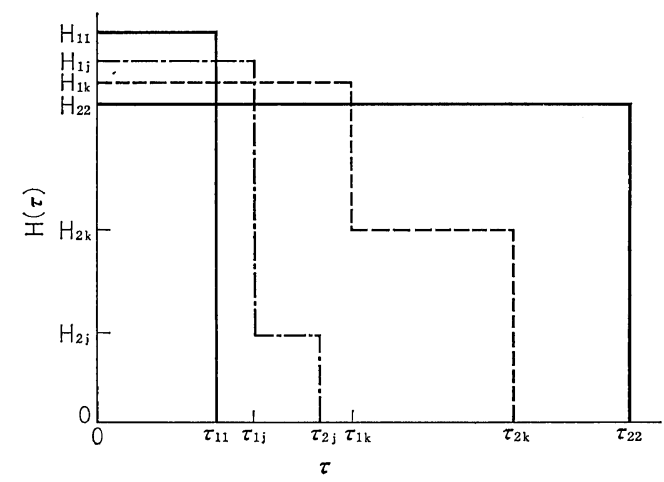

Figure 3. Addition of box-shaped relaxation spectra.

Table I. Blending law parameters

\begin{tabular}{|c|c|c|c|c|c|c|}
\hline$w_{2}$ & $V_{11}$ & $V_{22}$ & $\lambda_{11}$ & $\lambda_{22}$ & $\lambda_{22} / \lambda_{11}$ & $\sum_{i=1}^{2} V_{i i} \lambda_{i i}^{1 / 2}$ \\
\hline 0.000 & 1.00 & & 1.00 & & & \\
\hline 0.023 & 0.95 & 0.007 & 1.06 & 0.17 & 0.16 & 0.98 \\
\hline 0.049 & $0.9 \overline{0}$ & 0.0125 & 1.25 & 0.23 & $0.18 \underline{4}$ & 1.01 \\
\hline 0.089 & $0.8 \overline{5}$ & 0.024 & 1.35 & 0.27 & 0.20 & 1.00 \\
\hline 0.209 & $0.8 \overline{0}$ & 0.067 & 1.80 & 0.45 & 0.25 & 1.12 \\
\hline 0.399 & 0.60 & $0.17 \underline{\underline{5}}$ & 2.40 & 0.70 & 0.29 & 1.08 \\
\hline 1.000 & & 1.000 & & 1.00 & & \\
\hline
\end{tabular}

Table II. Terminal properties of blends at $192^{\circ} \mathrm{C}$

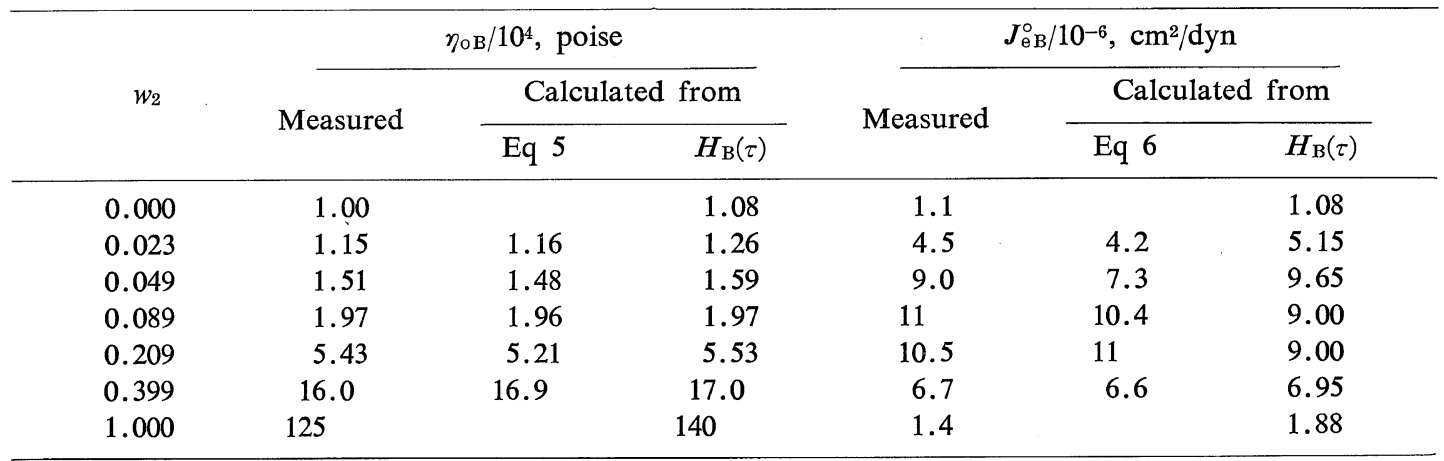




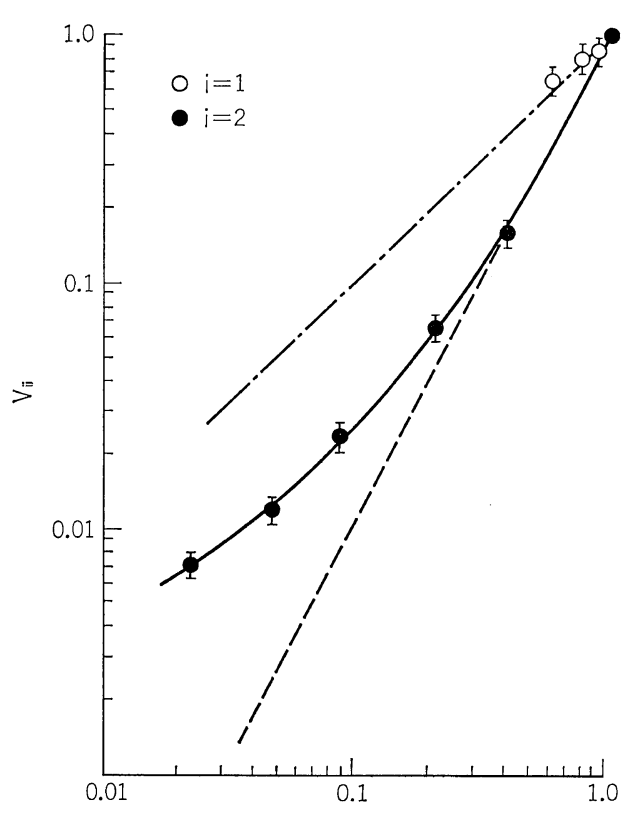

Figure 4. The dependence of the effective weighting factors $v_{i i}$ on the weight fractions $w_{i}$ of the high- $(i=2)$ and low $(i=1)-\mathrm{MW}$ components of the 411000/97200-blend series: ---, $w_{i}{ }^{2} ;-\cdot-, w_{i}{ }^{2}$.

Table III. Experimental and calculated $V_{11}$

\begin{tabular}{ccc}
\hline \multirow{2}{*}{$w_{2}$} & \multicolumn{2}{c}{$V_{11}$} \\
\cline { 2 - 3 } & Measured & Eq 5 \\
\hline 0.023 & $0.9 \underline{5}$ & 0.94 \\
0.049 & $0.9 \underline{0}$ & 0.92 \\
0.089 & $0.8 \underline{5}$ & 0.83 \\
0.209 & $0.8 \underline{0}$ & 0.80 \\
0.399 & $0.6 \underline{0}$ & 0.67 \\
\hline
\end{tabular}

determined by the maximum and minimum values of the height of the shoulders and represent the possible uncertainties in the calculated weighting factors. The dot-dash-dot line is the behavior that would be expected if $V_{i i}=$ $w_{i}$, the condition of the simple blending law proposed by Ninomiya ${ }^{1}$. The dashed line corresponds to $V_{i i}=w_{i}{ }^{2}$. The observed $V_{22}$ are proportional to $w_{2}^{2}$ at large $w_{2}$. However, signifcant deviations from this quadratic behavior are observed at small $w_{2}$. Note that unlike $V_{22}$, the weighting factors for the low-MW component $V_{11}$, are approximately equal to the weight fractions of the low component, $w_{1}$. Thus, even though there is a great deal of uncertainty in the determination of $V_{11}$, the sum of $V_{11}$ and $V_{22}$ is not one. This observation is confirmed by calculating $V_{11}$ from eq 5 and the measured values of $V_{22}, \lambda_{22}, \lambda_{11}$, and the experimental $\eta_{0 \mathrm{~B}}$. The calculated $V_{11}$, listed in Table III, are consistant with the measured values. Thus, if the simple blending law is to describe the interactions of high-MW polymers, the resulting blending law is not symmetric in the weighting factors of the components.

\section{Shift Factors}

Implicit in the determination of $V_{i i}$ is the necessity of shifting the components spectra by an amount $\lambda_{i i}$. The shift factors represent the ratios of a molecular species relaxation time in a blend to that time in the pure component. Relaxation times in polymers are controlled by the elastic properties and the viscous drag forces in the melt. In blends of components with similar elastic properties the shift factors are expected to be related to the same MW averages which control the viscosity of the melt, $M_{w}$. This is also implied by the simple blending law. If $V_{i i}=w_{i}$ and all the viscosities are simply related to $M_{w}$, then eq 5 implies that $\lambda_{i i}$ is a function of $M_{w}$. This restriction is relaxed as $V_{i i}$ becomes a more complicated function of $w_{i}$, i.e., as the elastic effects of entanglements become important. The experimental shift factors are more listed in Table I along with $\lambda_{22} / \lambda_{11}$. This ratio is seen to increase with $w_{2}$. Examination of the data shows that while $\lambda_{11}$ can be represented by a power of $M_{w}, \lambda_{22}$ depends on a higher moment of the MWD. A correlation of $\lambda_{11}$ with $M_{w}$ and $\lambda_{22}$ with $M_{z}$ is presented in Figure 5. The asymmetry of this dependence reflects the differences between the functional forms of $V_{11}$ and $V_{22}$.

If the blending law is to apply to the entire relaxation spectrum of the components, that is to the relaxation mechanisms of short molecular segments as well as those associated with entanglements, then the exferimental parameters must be consistent with the response of $H(\tau)$ in the transition region. The Rouse theory ${ }^{17}$ predicts that $H(\tau)$ is proportional to $\tau^{-1 / 2}$ in the transition region. For a given polymer, the isofree volume transition region response is inde- 

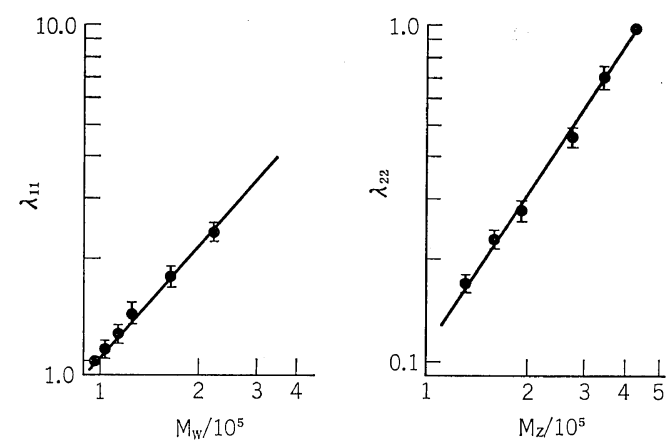

Figure 5. The dependence of the shift factor $\lambda_{11}$ and $\lambda_{22}$ on the weight and $Z$-average molecular weights of the blends.

pendent of MW and MWD. ${ }^{14}$ This has been demonstrated for polystyrene ${ }^{10}$. The simple blending law reduces each component's $\tau^{-1 / 2}$ region response by $V_{i i}$. In addition, the shift factors effectively change the contribution to $H_{\mathrm{B}}(\tau)$ at a given $\tau$ by $\lambda^{1 / 2}$. Therefore, if the transition region response is to be independent of MWD,

$$
\sum_{i=1}^{2} V_{i i} \lambda_{i i}^{1 / 2}=1
$$

The experimental values of this sum, listed in Table I, prove that the simple blending law may be applied to the entire VE spectrum of high MW polystyrene blends. Equation 9 also explains why the sum of the experimental weighting factors is not equal to one.

Relationships between the shift factors may be obtained from Figure 3. Define the ratio of the maximum relaxation times of the components and the ratio of those in the blend as

$$
L_{21}=\tau_{22} / \tau_{11} ; l_{21 k}=\tau_{2 k} / \tau_{1 k}
$$

Then from eq 7 and 8

$$
\lambda_{22} / \lambda_{11}=l_{21 k} / L_{21}
$$

The choice of the components defines $L_{21}$, which is a constant for any blend series. For the box shaped relaxation spectra in Figure 3, $\eta_{0 i i}=H_{i i} \tau_{i i}$. Then $L_{21}$ is proportional to the ratio of the viscosities of the components and thus to a power of the ratio of the molecular weights of the blended species. The width of the secondary plateau is proportional to $l_{12 k}$. Thus the ratio of the shift factors is a measure of the width of the secondary plateau relative to the difference between the components terminal response. Figure 1 shows that the width of the shoulder on $H_{\mathrm{B}}(\tau)$ decreases with the concentration of the high-MW component, in accordance with the observed dependence of $\lambda_{22} / \lambda_{11}$ (see Table I). This conflicts with Ninomiya and Ferry's ${ }^{6}$ observation that $\lambda_{22} / \lambda_{11}$ is a constant for high MW polystyrene blends. However, in that study, the $V_{i i}$ were assumed to be the weight fractions of the components and the $\lambda_{i i}$ were determined from the experimental relaxation moduli. This definition of $V_{i i}$ would decrease the apparent compositional dependence of the ratio of the shift factors.

The observed compositional dependence of $\lambda_{22} / \lambda_{11}$ limits the general application of the blending laws. Ninomiya and Ferry ${ }^{6}$ have shown that the blending laws may be applied to blends of belends, if and only, if the ratio of the shift factors is independent of $w_{2}$ for a given ratio of MW's. This is demonstrated by the relaxation spectrum of blend $j$ in Figure 3. Sample $j$ may be considered to be either a blend of components 1 and 2 or an appropriate mixture of component 1 and blend $k$. If the blending laws apply to the latter case, the maximum relaxation time of blend $k$ is shifted by an amount $\lambda_{k j}=\tau_{2 k} / \tau_{2 j}$. But all parts of the spectrum must be shifted by the same amount, $\tau_{1 k} / \tau_{i j}=\tau_{2 k} / \tau_{2 j}$, which is equivalent to $l_{21 j}=l_{21 k}$. This states that the width of the secondary plateau must be a constant if the blending law is to apply to blends of blends, a condition which is not observed in the experimental data presented in Figure 1.

\section{PARAMETERS OF THE QUADRATIC- BLEDING LAW}

\section{Long-Time Response.}

The initial dependence of $V_{22}$ on $w_{2}{ }^{2}$ shown in Figure 4 supports the idea of a quadraticblending law. In addition, it may be argued that the deviation of $V_{22}$ from the quadratic behavior is the result of the contribution of the cross relaxation spectrum. To test this proposition it is necessary to calculate $H_{12}\left(\tau / \lambda_{12}\right)$.

If the cross term is to account for the observed deviations, the response of the shoulder of the relaxation spectrum of a blend must be composed of the sum of the quadratic term ${w_{2}}^{2} H_{22}(\tau)$ 
$\left.\lambda_{22}\right)$ and $2 w_{1} w_{2} H_{12}\left(\tau / \lambda_{12}\right)$. Then, since $w_{1}, w_{2}$, and $H_{22}(\tau)$ are defined, and $w_{1}{ }^{2} H_{11}\left(\tau / \lambda_{11}\right)$ is negligible, the experimental $H_{\mathrm{B}}(\tau)$ can be used to obtain the response of $H_{12}\left(\tau / \lambda_{12}\right)$ and the shift factor $\lambda_{22}$.

In the analysis of the simple-blending law it was found that the shoulder in $H_{\mathrm{B}}(\tau)$ could be described by $V_{22} H_{22}\left(\tau / \lambda_{22}\right)$. Then for $\tau$ corresponding to relaxation processes represented by the shoulder in $H_{\mathrm{B}}(\tau)$, the experimental data is related to the quadratic blending law by

$$
V_{22} \frac{H_{22}\left(\tau / \lambda_{22}^{*}\right)}{H_{22}\left(\tau / \lambda_{22}\right)}=w_{2}{ }^{2}+2 w_{1} w_{2} \frac{H_{12}\left(\tau / \lambda_{12}\right)}{H_{22}\left(\tau / \lambda_{22}\right)}
$$

where the experimentally measured shift factor is denoted by $\lambda_{22}^{*}$. The cross term, $H_{12}\left(\tau / \lambda_{12}\right)$ can be calculated from the experimental data once $\lambda_{22}$ is chosen. In general the shape of $H_{12}(\tau)$ will be different than the shape of $H_{22}(\tau)$ unless $\lambda_{22}=\lambda_{22}^{*}$. However, if $\lambda_{22}$ does not equal $\lambda_{22}^{*}$ the shape of $H_{12}(\tau)$ is a function of the composition of the blend (see eq 12). But the form of the blending law states that $H_{12}(\tau)$ is independent of $w_{2}$. Therefore $\lambda_{22}=\lambda_{22}^{*}$ and $H_{12}\left(\tau / \lambda_{12}\right) / H_{22}\left(\tau / \lambda_{22}\right)$ is a constant. Then, if the quadratic-blending law describes the blends long-time response, a plot

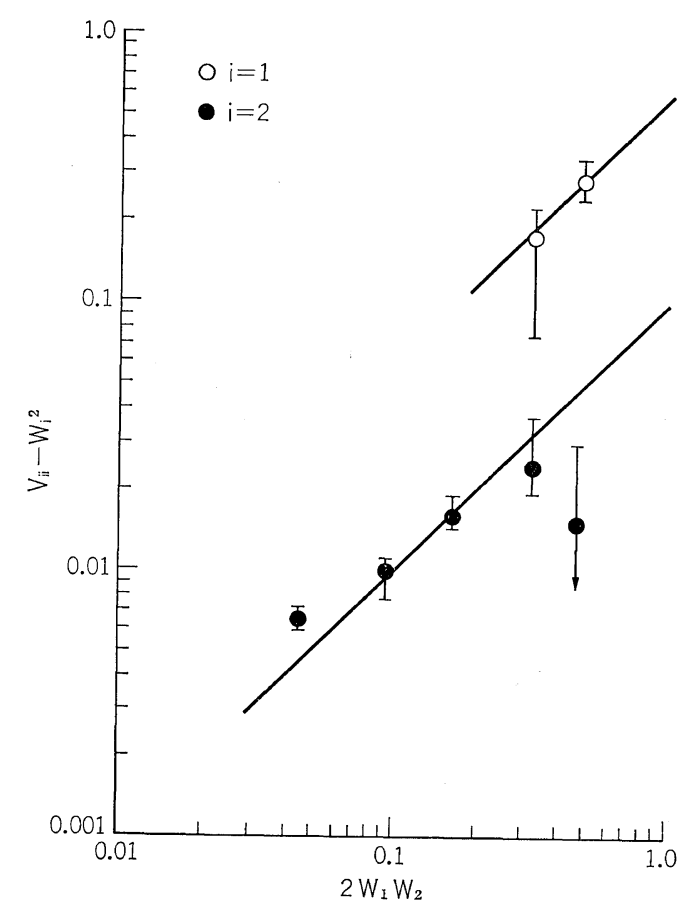

Figure 6. Calculation of the magnitude of the cross relaxation spectrum. of $\log \left(V_{22}-w_{2}^{2}\right)$ vs. $\log \left(2 w_{1} w_{2}\right)$ will produce a line with a unit slope. From this graph the the quantity $H_{12} / H_{22}$ can be evaluated. The data presented in Figure 6 give qualified support to the cross-term proposal. The line drawn with a unit slope indicates that the magnitude of $H_{12}$ in the long time region is is approximately onetenth that of $H_{22}$.

\section{Short-Time Response}

While the cross term, $H_{12}\left(\tau / \lambda_{12}\right)$ can be used to account for the difference between $V_{22}$ and $w_{2}{ }^{2}$; it does not explain the observed defendence of $V_{11}$ on $w_{1}$. The shape of the blends shorttime rubbery plateau response is experimentally independent of the compositional ratio (see Figure 1). Therefore, by the arguments developed above, the responses of $H_{11}\left(\tau / \lambda_{11}\right)$ and $H_{12}\left(\tau / \lambda_{12}\right)$ have similar shapes and, at short times, $\lambda_{12}=\lambda_{11}$. The ratio of the magnitude of these spectra may be calculated in a manner similar to eq 12 . The widely scattered results shown in Figure 6 imply that $H_{12} / H_{11} \approx 0.6$. Thus, since $H_{12} / H_{22} \approx 0.1$, the cross term, $H_{12}$ must be a two step function of $\tau$ (i.e., exhibit two plateaus). However, the experimentally observed compositional dependence of $\lambda_{22} / \lambda_{11}$ (Table I) states that the cross term cannot remain independent of $w_{2}$ and simultaneously be subject to the conditions that $\lambda_{12}=\lambda_{11}$ at low $\tau$ and $\lambda_{12}=\lambda_{22}$ at high $\tau$. Therefore the quadratic blending law with a single cross term (eq 2) does not describe the observed relaxation spectra.

It is important to note that Graessley's quadratic blending law is not subject to the above condition because it contains two cross terms, $H_{12}\left(\tau / \lambda_{12}\right)$ and $H_{21}\left(\tau / \lambda_{21}\right)$, which in general are not equal. In addition, the required similarity between the shapes of the cross terms and the component's spectra make Graessley's quadraticblending law equivalent to the simple-blending law with

$$
\begin{array}{ll}
v_{11}=w_{1}^{2}+w_{1} w_{2} H_{12} / H_{11}, & \lambda_{12}=\lambda_{11} \\
V_{22}=w_{2}^{2}+w_{1} w_{2} H_{21} / H_{22}, & \lambda_{21}=\lambda_{22}
\end{array}
$$

Thus, by definition, the two-term quadraticblending law describes the experimental longand short-time response of $H_{\mathrm{B}}(\tau)$.

The experimental conclusion that the simple and the quadratic blending laws are equivalent 
representations of $H_{\mathrm{B}}(\tau)$ is particularly surprising because the cross terms were originally introduced to account for the appearance of a set of relaxation times intermediate to the shifted spectra of the components ${ }^{10}$. This set of $\tau$ appears in the spectra of the $w_{2}=0.209$ and $w_{2}=0.399$ blends shown in Figure 1 as the broad transition between the main body and the shoulder of $H_{\mathrm{B}}(\tau)$. Note that this transition is more gradual than the rapid fall off in the terminal response of the 97200-MW component.

While this broad intermediate response seems to represent a new set of relaxation times, it can be shown that this region is just the sum of the relaxation spectra of the components. This is demonstrated in Figure 7 for the $w_{2}=$ 0.209 blend. The solid line is the measured

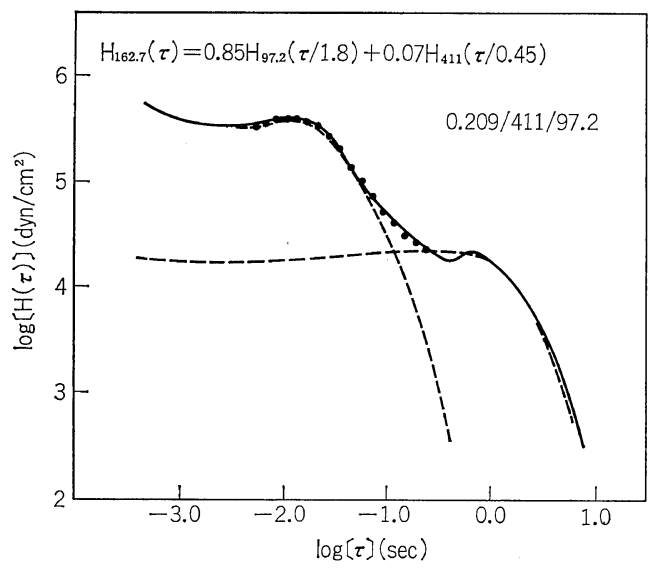

Figure 7. The simple-blending law construction of the relaxation spectrum of the $w_{2}=0.209$ blend from the sum $(\cdots)$ of the relaxation spectra (--) of the components.

$H_{\mathrm{B}}(\tau)$. The broken lines are the spectra of the two components, weighted and shifted by $V_{i i}$ and $\lambda_{i i}$. The solid points are the sum of these contributions. The shape of this intermediate region is controlled by the terminal response of $V_{11} H_{11}\left(\tau / \lambda_{11}\right)$ over the range of $\tau$ where it intersects $V_{22} H_{22}\left(\tau / \lambda_{22}\right)$. As $H_{11}(\tau)$ decreases, the slope of $H_{11}(\tau)$ decreases. Thus the contribution of $H_{11}\left(\tau / \lambda_{11}\right)$ to the intermediate time response decreases with the concentration of the higher-MW component. This accounts for the broad transition observed at large $w_{2}$ and the apparent lack of this response at small $w_{2}$.

\section{DISCUSSION}

The choice of a particular blending law depends on a proposed theoretical model's ability to calculate either the set of parameters, $V_{11}$ and $V_{22}$ or $H_{12} / H_{22}$ and $H_{21} / H_{22}$. At first it appears that the $V_{i i}$ are useful only in the calculation of $H_{21} / H_{22}$ and $H_{12} / H_{22}$ from experimental data. However, the $V_{i i}$ have particular significance by themselves. This may be seen from eq 4 . At low frequencies $G^{\prime \prime}$ is the stress on the sample. Then the $V_{i i}$ represent the contribution of molecular species $i$, to the total stress supported by the sample.

As an example, the simple blending law may be applied to the formalism developed by Fix$\operatorname{man}^{19}$ concerning the behavior of molecules in solution. According to Fixman ${ }^{19}$, the stress tensor of a flexible molecule in solution is

$$
\sigma=\left\langle\sigma_{0}\right\rangle+c \sum_{i} R_{i} \nabla_{i} U+1 / 2 c^{2}\left\langle R_{12} \nabla_{2} \bigvee\left(R_{12}\right)\right\rangle
$$

where $\sum R_{i} \nabla_{i} U$ is an average over the interactions between each segment and all other segments in the solution and $c$ is the concentration of the molecules in solution. The coefficient of the $c^{2}$ term takes into account interactions between separate molecules other than entanglements (i.e., excluded volume) through the potential $\bigvee\left(R_{12}\right)$ where $R_{12}$ is the vector distance between the molecules center of mass. Applications of Fixman's equation assume that the interaction potential $U$ is a pairwise additive function of the potential energy between segments. Williams ${ }^{20}$ points out that this assumption fails in concentrated solutions where it is necessary to take into account three body cooperative interactions. Such a treatment would add a $c^{3}$ term to the stress tensor, physically corresponding to entanglement interactions. If individual molecules in in a melt can be considered to be molecules in a very concentrated solution and if the stress contributions can be treated in this manner the experimental $V_{i i}$ should be representable by a cubic equation in the individual weight fractions, $w_{i}$. The coefficients of the $c, c^{2}$, and $c^{3}$ terms would then represent the intramolecular, the intermolecular and the entanglement interaction contribution to the total stress. As the size (i.e., MW ) and concentration of the individual components increased, the major contribution 
to the stress would shift from a primary dependence on $c$, to $c^{2}$, and $c^{3}$. The weighting factor of the low-MW species in a blend would then have a different functional dependence than the $V_{i i}$ of the high-MW components.

The systematic deviation of the data in Figure 6 from the first power dependence on $w_{1} w_{2}$ suggests that the $V_{i i}$ may be better represented by a cubic equation in $w_{2}$ than by the quadraticblending law. The results of a least squares regressional analysis are given by eq 15 .

$$
V_{22}=0.21 w_{2}+0.42 w_{2}{ }^{2}+0.37 w_{2}{ }^{3}
$$

In the context of the previous discussion this suggests that, for $M=411000$, the entanglement contribution to the stress is of the same order as that supported by the intermolecular interactions and about twice that of the intramolecular term.

\section{REFERENCES}

1. K. Ninomiya, J. Colloid Sci., 14, 527 (1959).

2. D. C. Bogue, T. Masuna, Y. Einaga, and S. Onogi, Polymer J., 1, 563 (1970).

3. W. W. Graessley, J. Chem. Phys., 54, 5143 (1971).

4. K. Ninomiya, J. Colloid Sci., 17, 759 (1962).

5. K. Ninomiya, J. D. Ferry and Y. Ôyanagi, $J$.
Phys. Chem., 67, 2297 (1963).

6. K. Ninomiya and J. D. Ferry, J. Colloid Sci., 18, 421 (1963).

7. K. Ninomiya and G. Yasuda, Rubber Chem. Technol., 40, 493 (1967).

8. K. Ninomiya and J. D. Ferry, J. Macromol. Sci.-Phys., B3(2), 237 (1969).

9. W. M. Prest, Jr., J. Polym. Sci., Part A-2, 8, 1897 (1970).

10. T. Masuda, K. Kitagawa, T. Inoue and $\mathbf{S}$. Onogi, Macromolecules, 3, 116 (1970).

11. N. J. Mills and A. Nevin, J. Polym. Sci., Part A-2, 9, 267 (1971).

12. W. M. Prest, Jr. and R. S. Porter, submitted to Polymer J., 4, 154 (1973).

13. B. Gross, "Mathematical Structure of the Theories of Viscoelasticity", Hermann, et Cie., Paris, 1953.

14. J. D. Ferry, "Viscoelastic Properties of Polymers", John Wiley, New York, N. Y., 1961, 1970.

15. W. M. Prest, Jr., Ph. D. Thesis, University of Massachusett, Amherst, Mass., 1972.

16. N. W. Tschoegl, submitted to Trans. Soc. Rheo$\log y$ (ref. 14, pp 92 and 94).

17. P. E. Rouse, J. Chem. Phys., 21, 1272 (1953).

18. A. J. Chompff and J. A. Duiser, J. Chem. Phys., 45, 1505 (1966).

19. M. J. Fixman, ibid., 42, 3831 (1965).

20. M. C. Williams, A.I.Ch.E.-J., 12, 1064 (1966). 